\title{
Break on Through to the Single Side
}

\author{
Dilip B. Madan*and Wim Schoutens ${ }^{\dagger}$
}

26th of July 2007

*Robert H. Smith School of Business, Van Munching Hall 4409, University of Maryland, College Park MD, 20742, E-mail: dbm@rhsmith.umd.edu

${ }^{\dagger}$ K.U.Leuven, Department of Mathematics, Celestijnenlaan 200 B 54, B-3001 Leuven, Belgium. E-mail: wim@schoutens.be 


\begin{abstract}
We employ a Lévy process subject to only negative jumps to describe the motion of asset values. This specification permits fast computation of first passage probabilities. As a result we are able to calibrate all CDS curves for the 125 iTraxx underliers weekly and develop a time series for the implied parameter values. A variety of models are investigated for the process: gamma, inverse Gaussian and the one sided CGMY, here referred to as CMY.
\end{abstract}




\section{Introduction}

There has been an exponential growth in credit derivatives in recent years. Culminating with a developed liquid market in Credit Default Swaps (CDS), for example the 125 names in the iTraxx and CDX indices.

There are basically two main classes of models for credit pricing: intensity based models and structural models. This manuscript proposes a structural model for the asset value process with default occurring the first time asset value breaches a low barrier. This barrier typically corresponds to the recovery value of the firm's debt. In this regard, the model shares structural characteristics of the Black-Cox model [2].

Most available structural models, like the Black-Cox model, use a stochastic process with continuous paths to model the firm's asset value. For example, the Black-Cox model is built upon the hypothesis that the asset value is described by a Geometric Brownian motion (Black-Scholes model). Because, a Brownian motion has continuous sample paths, in the Black-Cox setting, an event of default occurs exactly when the asset value of the firm hits the deterministic barrier. It is never the case that the firm's value overshoots the default level. Moreover, it is furthermore well known that due to the continuous path nature of Brownian motion and the underlying Normal distribution, the model cannot represent a realistic behavior of short term default probabilities. Indeed, the Brownian motion needs, because of its continuous paths, a substantial amount of time to reach a low barrier under a realistic volatility assumption. From a technical point of view, this implies that default times are in some sense predictable. In a situation where one is far away from the barrier, one is sure no default will occur the next instant. In reality however, default can occur at any time (cfr. Parmalat event). Moreover, empirical evidence suggests that the underlying $(\log )$ normal distribution does not accurately describe the law of motion in asset values. Default events are most of the time triggered by shocks (which are represented by downward jumps in the asset value). So any process modeling the stochastic nature of losses should reasonable include jumps and needs to incorporate skewness in the underlying return distribution. Lévy processes, incorporate exactly these jumps and asymmetry features and have proven already their modeling abilities in other settings [7] like equity and fixed income and have recently found their way into credit risk. This paper illustrates this by proposing such a Lévy based model that moreover turns out to be very tractable.

More precisely, we build a model, where default is indeed triggered by asset value crossing a very low barrier, and we further assume that the asset value process is described by an exponential of a Lévy process with an upward drift and only downward jumps. Such processes are called spectrally negative or one sided negative jump Lévy processes with positive drift. As such, we take into account both asymmetry and fat-tail behavior.

One could argue that in contrast to the behavior of stock prices where possibly both up and down jumps are present, a firm tries to follow a steady growth (up trend drift) but is exposed to shocks (negative jumps). It thus seems apriori 
reasonable to model the underlying firm's value in a default model by a process with a positive drift subject to negative jumps. In this work we will focus purely on the one-sided situation. In contrast to double sided Lévy processes, which were for example applied by Cariboni and Schoutens [3], we do not need to solve a rather time-consuming Partial-Integro-Differential Equation (PIDE) in order to obtain the default probabilities over time. Indeed, the advantage of only allowing negative jumps in the driving Lévy process is the fact that we can calculate extremely fast the first passage time distributions (default probabilities) by exploiting the remarkable Wiener-Hopf factorization in combination with results by Rogers [5] and by performing a very fast double Laplace transform inversion. In contrast to the double sided situation, where the solution of the PIDE takes typically a couple of seconds on an ordinary computer, the double Laplace transform inversion can be performed within a fraction of a second.

Default probabilities are the essential quantities in the calculation of single name CDSs. These financial instruments are the most widely used credit derivatives and provide protection against defaults of individual firm's and have seen an explosive growth over the last decade. They are unfunded securities designed to transfer the credit exposure of a reference entity between parties and to allow them to take positions in both directions according to their specific needs. They are nowadays liquidly traded for thousands of underlying companies. An illustration of the success are the iTraxx and CDX indices. The most popular are iTraxx Europe main index composed of the most important 125 CDS referencing European investment grade credits and the CDX.NA.IG index containing in a similar manner 125 CDSs of North American companies.

The fact that the calculation of default probabilities can be done very fast, makes the calibration of the model to markets possible and allows us to make a historical study of the model's performance for all iTraxx constituents. In a typical calibration, thousands of these default probabilities and there corresponding CDS quotes need to be calculated and hence fast computation of default probabilities are essential to the success of any model.

The calibration results are very satisfactory as is shown in a calibration exercise on historical prices of all the CDSs constituting the iTraxx. Calibration errors are in the order of 1 bp per quote. The historical calibration study also allows us to form a view on how the market prices moves of different sizes. We have focussed our attention on the 5, 10 and 20 percentage moves in asset value and report on the approximate daily arrival rates of a 5 percent move, the premium of 5 percent moves to 10 percent moves (the spread) and the relative spread, (the logarithm of the 10 to 5 percent market spread over the 20 to 10 percent market spread).

This paper is organized as follows. In Section 2, we set up the one-sided Lévy default model and work out the details of the calculation of default probabilities under the model. In Section 3, we overview some tractable examples based on some popular spectrally negative Lévy processes, like the Gamma, the IG and the CMY process. Section 4 deals with the pricing of CDSs under the proposed model and elaborates on the above mentioned historical calibration study. 


\section{One Sided Lévy Default Model}

\subsection{One Sided Firm's Value Process}

We will describe the asset value of the firm by a stochastic process $A=\left\{A_{t}, t \geq\right.$ $0\}$ and define the default event as the first crossing of some predetermined barrier $R$.

We work under the so-called one sided Lévy model in the sense that we assume that the risk-neutral firm's value follows an exponential of a spectrally negative Lévy process $X=\left\{X_{t}, t \geq 0\right\}$. Let us in general consider a spectrally negative Lévy process $Y=\left\{Y_{t}, t \geq 0\right\}$ without drift and set

$$
X_{t}=(r-\omega) t+Y_{t},
$$

where $\omega=\log \left(E\left[\exp \left(Y_{t}\right)\right]\right)$ in order to make the growth rate on the exponential of $X$ equal to the risk free interest rate. Then we have

$$
E\left[\exp \left(z X_{t}\right)\right]=\exp \left(t \psi_{X}(z)\right)
$$

where $\psi_{X}(z)$ is the Lévy exponent that by the Lévy-Khintchin representation has the form

$$
\psi_{X}(z)=\mu z+\int_{-\infty}^{0}\left(e^{z x}-1+z(|x| \wedge 1)\right) \nu(\mathrm{d} x),
$$

where $\mu=r-\omega$ is positive and the Lévy measure $\nu(\mathrm{d} x)$ satisfies the integrability condition

$$
\int_{-\infty}^{0}\left(|x|^{2} \wedge 1\right) \nu(\mathrm{d} x)<\infty
$$

In most practical cases the Lévy measure has a density and we write $\nu(\mathrm{d} x)=$ $m(x) \mathrm{d} x$ and refer to it as the Lévy density function. In the physical process, the Lévy density $m(x)$ represents the arrival rates of jumps of size $x$ in the process $X$. For the risk-neutral process, $m(x)$ is comparable to the market price of jumps of size $x$.

The risk neutral model for the firm's value process (standardized such that $\left.A_{0}=1\right)$ is given by

$$
A_{t}=\exp \left(X_{t}\right), \quad A_{0}=1 .
$$

Then default is defined to occur the first time when

$$
A_{t}=\exp \left(X_{t}\right) \leq R
$$

or equivalently if

$$
X_{t} \leq \log (R) .
$$

Let us denote by $P_{\text {surv }}(t)$ the risk-neutral probability of survival or no-default between 0 and $t$ :

$$
\begin{aligned}
P_{\text {surv }}(t) & =P_{Q}\left(X_{s}>\log (R), \text { for all } 0 \leq s \leq t\right) \\
& =P_{Q}\left(\inf _{0 \leq s \leq t} X_{s}>\log (R)\right) \\
& =P_{Q}\left(\underline{X}_{t}>\log (R)\right)
\end{aligned}
$$


where the subindex $Q$ refers to the fact that we are working in a risk-neutral setting. Next, we focus on how to calculate the above survival probability. The method will be based on the Wiener-Hopf factorization identity together with a double Laplace transform inversion method.

\subsection{First Passage Times for Spectrally Negative Lévy Pro- cesses}

In this section we will detail the technique to calculate very efficiently $P_{\text {surv }}(t)$, which actually boils down to the calculation of a first passage time (at a low level) of the underlying Lévy process.

In order to apply the inversion methodology described below, what is needed is that for large $z$ we have that

$$
\frac{\psi(z)-\mu z}{z} \rightarrow 0 .
$$

Equivalently this means that

$$
\lim _{z \rightarrow \infty} \frac{1}{z} \int_{-\infty}^{0}\left(e^{z x}-1+z(|x| \wedge 1)\right) \nu(\mathrm{d} x)=0 .
$$

The condition can be checked out in every specific application.

Since default is triggered by the crossing of a low barrier, or equivalently by the point where the running minimum will cross that level, essential in the sequel will be the distribution of the running maximum and minimum of the Lévy process. Define the running maximum and minimum of the Lévy process $X=\left\{X_{t}, t \geq 0\right\}$ by

$$
\begin{aligned}
& \bar{X}_{t}=\sup _{u \leq t} X_{u} \\
& \underline{X}_{t}=\inf _{u \leq t} X_{u}
\end{aligned}
$$

Let $T_{\lambda}$ and independent exponential random variable with parameter $\lambda$. Then Laplace transform of the process taken at an exponential time is given by

$$
E\left[\exp \left(z X_{T_{\lambda}}\right)\right]=\frac{\lambda}{\lambda-\psi(z)} .
$$

For this expression we have the remarkable factorization (valid for general Lévy processes):

$$
\begin{aligned}
\frac{\lambda}{\lambda-\psi(z)} & =\psi_{\lambda}^{+}(z) \psi_{\lambda}^{-}(z) \\
& =E\left[\exp \left(z \bar{X}\left(T_{\lambda}\right)\right)\right] E\left[\exp \left(z \underline{X}\left(T_{\lambda}\right)\right)\right]
\end{aligned}
$$

In other words, the Laplace transform of the process $X$ at an independent exponential time factorizes into the Laplace transforms of the running minimum and 
the running maximum taken at exponential time. This is the so-called WienerHopf factorization; $\psi_{\lambda}^{+}(z)$ and $\psi_{\lambda}^{-}(z)$ are called the (left and right) Wiener-Hopf factors. These factors are unique.

Moreover, classical Lévy process theory (see for example [1, Corollary 2], [6] or [4]) shows that for a spectrally negative processes the right Wiener-Hopf factor equals

$$
\psi_{\lambda}^{+}(z)=\frac{\beta^{*}}{\beta^{*}-z}
$$

where $\beta^{*}$ is a constant depending on $\lambda: \beta^{*}=\beta^{*}(\lambda)$, and that $\beta^{*}$ is a solution to

$$
\psi(\beta)=\lambda
$$

In other words the running maximum at an exponential time (with parameter $\lambda)$ is exponentially distributed with parameter $\beta^{*}=\psi^{[-1]}(\lambda)$.

It follows that

$$
\psi_{\lambda}^{-}(z)=\frac{\lambda}{\lambda-\psi(z)} \frac{\beta^{*}-z}{\beta^{*}} .
$$

Now note that by partial integration

$$
\begin{aligned}
\psi_{\lambda}^{-}(z) & =\int_{t=0}^{t=\infty} \int_{x=-\infty}^{x=0} \lambda \exp (-\lambda t) \exp (z x) f_{\underline{X}_{t}}(x) \mathrm{d} x \mathrm{~d} t \\
& =\int_{t=0}^{t=\infty} \int_{x=-\infty}^{x=0} \lambda \exp (-\lambda t) \exp (z x) z P\left(\underline{X}_{t}>x\right) \mathrm{d} x \mathrm{~d} t \\
& =\lambda z \int_{t=0}^{t=\infty} \int_{x=-\infty}^{x=0} \exp (-\lambda t) \exp (z x) P\left(\underline{X}_{t}>x\right) \mathrm{d} x \mathrm{~d} t \\
& =\frac{\lambda}{\lambda-\psi(z)} \frac{\beta^{*}-z}{\beta^{*}}
\end{aligned}
$$

Let us now focus on the time at which the running minimum crosses for the first time a barrier $x$, i.e.

$$
H_{x}=\inf \left\{t: X_{t}<x\right\}
$$

then

$$
\begin{aligned}
f(t, x) & =P\left(H_{-x}>t\right) \\
& =P\left(\underline{X}_{t}>-x\right) .
\end{aligned}
$$

Hence, we observe that the double Laplace transform of $f$

$$
\begin{aligned}
g(\lambda, z) & =\int_{t=0}^{t=\infty} \int_{x=0}^{x=\infty} \exp (-\lambda t-z x) f(t, x) \mathrm{d} x \mathrm{~d} t \\
& =\int_{t=0}^{t=\infty} \int_{x=-\infty}^{x=0} \exp (-\lambda t+z x) P\left(\underline{X}_{t}>x\right) \mathrm{d} x \mathrm{~d} t \\
& =\frac{\beta^{*}(\lambda)-z}{(\lambda-\psi(z)) \beta^{*}(\lambda) z}
\end{aligned}
$$


We may then write for real numbers

$$
\begin{aligned}
z & =z_{1}-\mathrm{i} z_{2} \\
\lambda & =\lambda_{1}-\mathrm{i} \lambda_{2}
\end{aligned}
$$

that

$$
g\left(\lambda_{1}-\mathrm{i} \lambda_{2}, z_{1}-\mathrm{i} z_{2}\right)=\int_{x=0}^{x=\infty} \int_{t=0}^{t=\infty} \exp \left(-\lambda_{1} t+\mathrm{i} \lambda_{2} t-z_{1} x+\mathrm{i} z_{2} x\right) f(t, x) \mathrm{d} t \mathrm{~d} x
$$

so that

$$
g\left(\lambda_{1}-\mathrm{i} \lambda_{2}, z_{1}-\mathrm{i} z_{2}\right)
$$

is the double Fourier transform of

$$
\exp \left(-\lambda_{1} t-z_{1} x\right) f(t, x)
$$

So by the inverse Fourier transform we have that

$\exp \left(-\lambda_{1} t-z_{1} x\right) f(t, x)=\frac{1}{(2 \pi)^{2}} \int_{\lambda_{2}=-\infty}^{\lambda_{2}=\infty} \int_{z_{2}=-\infty}^{z_{2}=\infty} \exp \left(-\mathrm{i} \lambda_{2} t-\mathrm{i} z_{2} x\right) g\left(\lambda_{1}-\mathrm{i} \lambda_{2}, z_{1}-\mathrm{i} z_{2}\right) \mathrm{d} z_{2} \mathrm{~d} \lambda_{2}$

or equivalently we may write

$$
\begin{aligned}
f(t, x) & =\frac{1}{(2 \pi)^{2}} \int_{\lambda_{2}=-\infty}^{\lambda_{2}=\infty} \int_{z_{2}=-\infty}^{z_{2}=\infty} \exp \left(\left(\lambda_{1}-\mathrm{i} \lambda_{2}\right) t+\left(z_{1}-\mathrm{i} z_{2}\right) x\right) g\left(\lambda_{1}-\mathrm{i} \lambda_{2}, z_{1}-\mathrm{i} z_{2}\right) \mathrm{d} z_{2} \mathrm{~d} \lambda_{2} \\
& =-\frac{1}{(2 \pi)^{2}} \int_{\Gamma_{1}} \int_{\Gamma_{2}} \exp (\lambda t+z x) g(\lambda, z) \mathrm{d} \lambda \mathrm{d} z \\
& =-\frac{1}{(2 \pi)^{2}} \int_{\Gamma_{1}} \int_{\Gamma_{2}} \exp (\lambda t+z x) \frac{\beta^{*}(\lambda)-z}{(\lambda-\psi(z)) \beta^{*}(\lambda) z} \mathrm{~d} \lambda \mathrm{d} z
\end{aligned}
$$

where the contour $\left.\Gamma_{1}=\left\{\lambda_{1}+\mathrm{i} \lambda_{2} \mid \lambda_{2}=-\infty \cdots+\infty\right)\right\}$ and the contour $\Gamma_{2}=$ $\left.\left\{z_{1}+\mathrm{i} z_{2} \mid z_{2}=-\infty \cdots+\infty\right)\right\}$.

Performing this double transform gives us directly $f(t, x)$ the probability that the minimum stays above negative $x$ in $t$ units of time. There is no problem with $z$ or the contour $\Gamma_{2}$ but for the contour $\Gamma_{1}$ and $\lambda=\lambda_{1}-\mathrm{i} \lambda_{2}$ we have to solve the complex equation

$$
\psi(\beta)=\lambda_{1}-\mathrm{i} \lambda_{2}
$$

for the right value of $\beta^{*}$. This can be quite a difficulty and renders the solution relatively intractable.

The suggestion by Rogers in [5] is to alter the contour $\Gamma_{1}$ to the contour

$$
\Gamma_{0}=\psi\left(\Gamma_{1} / \mu\right),
$$

which is allowed if the condition (2) is satisfied. The underlying reason is that on this new contour, solving for $\beta$ is easy. Indeed for a value $\psi(\lambda / \mu)=\psi\left(\left(\lambda_{1}-\right.\right.$ $\left.\left.\mathrm{i} \lambda_{2}\right) / \mu\right) \in \Gamma_{0}$, we have

$$
\psi(\beta)=\psi(\lambda / \mu)
$$


and we have the solution

$$
\beta=\lambda / \mu
$$

Defining

$$
h(\lambda)=\psi(\lambda / \mu)
$$

we then write

$$
\begin{aligned}
f(t, x) & =-\frac{1}{(2 \pi)^{2}} \int_{\Gamma_{1}} \int_{\Gamma_{2}} \exp (h(\lambda) t+z x) g(h(\lambda), z) \mathrm{d} h(\lambda) \mathrm{d} z \\
& =-\frac{1}{(2 \pi)^{2}} \int_{\Gamma_{0}} \int_{\Gamma_{2}} h^{\prime}(\lambda) \exp (h(\lambda) t+z x) g(h(\lambda), z) \mathrm{d} \lambda \mathrm{d} z \\
& =-\frac{1}{(2 \pi)^{2}} \int_{\Gamma_{0}} \int_{\Gamma_{2}} h^{\prime}(\lambda) \exp (h(\lambda) t+z x) \frac{\beta^{*}(h(\lambda))-z}{(h(\lambda)-\psi(z)) \beta^{*}(h(\lambda)) z} \mathrm{~d} \lambda \mathrm{d} z \\
& =-\frac{1}{(2 \pi)^{2}} \int_{\Gamma_{0}} \int_{\Gamma_{2}} h^{\prime}(\lambda) \exp (h(\lambda) t+z x) \frac{(\lambda / \mu)-z}{(h(\lambda)-\psi(z))(\lambda / \mu) z} \mathrm{~d} \lambda \mathrm{d} z .
\end{aligned}
$$

The required double integral is approximated for fixed $t$ and $x$, by the double sum following Abate and Whitt,

$$
\begin{aligned}
S_{N}= & \frac{h_{1} h_{2}}{4 \pi^{2}} \sum_{n=-N}^{N} \sum_{m=-N}^{N} h^{\prime}\left(a_{1}+i n h_{1}\right) g\left(h\left(a_{1}+i n h_{1}\right), a_{2}+i m h_{2}\right) \\
& \times \exp \left\{t h\left(a_{1}+i n h_{1}\right)+x\left(a_{2}+i m h_{2}\right)\right\}
\end{aligned}
$$

It is suggested that we take

$$
\begin{aligned}
a_{1} & =\frac{A_{1}}{2 t l_{1}} \\
a_{2} & =\frac{A_{2}}{2 x l_{2}} \\
h_{1} & =\frac{\pi}{t l_{1}} \\
h_{2} & =\frac{\pi}{x l_{2}} \\
A_{1} & =A_{2}=22 \\
l_{1} & =l_{2}=1 \\
M & =15 \\
N & =12
\end{aligned}
$$

Finally one performs an Euler summation

$$
f(t, x) \stackrel{=}{=} \sum_{k=0}^{M} 2^{-M}\left(\begin{array}{c}
M \\
k
\end{array}\right) S_{N+k}
$$

where we use $M=15$ and $N=12$. 


\section{Examples}

\subsection{The Gamma Process}

The density function of the Gamma distribution $\operatorname{Gamma}(a, b)$ with parameters $a>0$ and $b>0$ is given by:

$$
f_{\text {Gamma }}(x ; a, b)=\frac{b^{a}}{\Gamma(a)} x^{a-1} \exp (-x b), \quad x>0 .
$$

The characteristic function is given by:

$$
\phi_{\text {Gamma }}(u ; a, b)=(1-\mathrm{i} u / b)^{-a}, \quad u \in \mathbb{R} .
$$

Clearly, this characteristic function is infinitely divisible. The Gamma-process $G=\left\{G_{t}, t \geq 0\right\}$ with parameters $a, b>0$ is defined as the stochastic process which starts at zero and has stationary, independent Gamma-distributed increments. More precisely, the time enters in the first parameter: $G_{t}$ follows a $\operatorname{Gamma}(a t, b)$ distribution.

The Lévy density of the Gamma process is given by

$$
m(x)=a \exp (-b x) x^{-1}, \quad x>0 .
$$

The properties of the $\operatorname{Gamma}(a, b)$ distribution given in Table 1 can easily be derived from the characteristic function.

\begin{tabular}{|c|c|}
\hline & $\operatorname{Gamma}(a, b)$ \\
\hline mean & $a / b$ \\
variance & $a / b^{2}$ \\
skewness & $2 / \sqrt{a}$ \\
kurtosis & $3(1+2 / a)$ \\
\hline
\end{tabular}

Table 1: Mean, variance, skewness and kurtosis of the $\operatorname{Gamma}(a, b)$ distribution.

Let us start with a Gamma-process $G=\left\{G_{t}, t \geq 0\right\}$ with parameters $a>0$ and $b>0$. As driving Lévy process (in a risk neutral setting), we then take:

$$
X_{t}=\mu t-G_{t}, \quad t \in[0,1],
$$

where in this case $\mu=r-\log (\phi(-i))=r+a \log \left(1+b^{-1}\right)$. We hence have a deterministic up-trend and negative shock are coming from a Gamma process. We refer to this model as the Shifted Gamma model $(S G)$.

The characteristic exponent in this case is available in closed form. More precisely,

$$
\psi(z)=\mu z-a \log \left(1+\frac{z}{b}\right) .
$$

Note further that

$$
h(\lambda)=\lambda-a \log \left(1+\frac{\lambda}{\mu b}\right) \text { and } h^{\prime}(\lambda)=1-\frac{a}{\mu b+\lambda} .
$$




\subsection{The IG Process}

The Inverse Gaussian $\operatorname{IG}(a, b)$ law with parameters $a>0$ and $b>0$ has characteristic function:

$$
\phi_{I G}(u ; a, b)=\exp \left(-a\left(\sqrt{-2 \mathrm{i} u+b^{2}}-b\right)\right), \quad u \in \mathbb{R} .
$$

The IG-distribution is infinitely divisible and we define the IG-process $I=$ $\left\{I_{t}, t \geq 0\right\}$ with parameters $a, b>0$ as the process which starts at zero, has independent and stationary IG-distributed increments, and such that:

$$
\mathbb{E}\left[\exp \left(\mathrm{i} u I_{t}\right)\right]=\phi_{I G}(u ; a t, b)=\exp \left(-a t\left(\sqrt{-2 \mathrm{i} u+b^{2}}-b\right)\right), \quad u \in \mathbb{R}
$$

meaning that $I_{t}$ follows a $\operatorname{IG}(a t, b)$ distribution.

The density function of the $\operatorname{IG}(a, b)$ law is explicitly known:

$$
f_{I G}(x ; a, b)=\frac{a}{\sqrt{2 \pi}} \exp (a b) x^{-3 / 2} \exp \left(-\left(a^{2} x^{-1}+b^{2} x\right) / 2\right), \quad x>0 .
$$

The Lévy density of the IG process is given by

$$
m(x)=\frac{a}{\sqrt{2 \pi}} \exp \left(-\frac{1}{2} b^{2} x\right) x^{-3 / 2}, \quad x>0 .
$$

\begin{tabular}{|c|c|}
\hline & $\mathrm{IG}(a, b)$ \\
\hline mean & $a / b$ \\
variance & $a / b^{3}$ \\
skewness & $3 / \sqrt{a b}$ \\
kurtosis & $3\left(1+5(a b)^{-1}\right)$ \\
\hline
\end{tabular}

Table 2: Mean, variance, skewness and kurtosis of the $\operatorname{IG}(a, b)$ distribution.

The characteristics given in Table 2 can easily be obtained.

Let us start with a unit variance IG-process $I=\left\{I_{t}, t \geq 0\right\}$ with parameters $a>0$ and $b>0$. In our model, we then take:

$$
X_{t}=\mu t-I_{t}, \quad t \in[0,1] .
$$

where in this case $\mu=r-\log (\phi(-i))=r+a\left(\sqrt{2+b^{2}}-b\right)$. We hence have again a deterministic up-trend and negative shock are now coming from a inverse Gaussian process. We refer to this model as the Shifted IG model (SIG).

The characteristic exponent in this case is available in closed form. More precisely,

$$
\psi(z)=\mu z-a\left(\sqrt{2 z+b^{2}}-b\right) .
$$

Note further that

$$
h(\lambda)=\lambda-a\left(\sqrt{2 \lambda \mu^{-1}+b^{2}}-b\right) \text { and } h^{\prime}(\lambda)=1-\frac{a}{\mu}\left(2 \lambda \mu^{-1}+b^{2}\right)^{1 / 2} .
$$




\subsection{The CMY Process}

The characteristic function of the CMY distribution $\operatorname{CMY}(C, M, Y)$ with parameters $C, M>0$ and $Y<1$ is given by:

$$
\phi_{C M Y}(u ; C, M, Y)=\exp \left(C \Gamma(-Y)\left((M-\mathrm{i} u)^{Y}-M^{Y}\right)\right) .
$$

Note that the CMY distribution can be casted sometimes also referred to (with an other parameter convention) as a Tempered Stable distribution.

The Lévy measure of the CMY process is given by:

$$
m(x)=C \exp (-M x) x^{-1-Y}, \quad x>0 .
$$

Observe from the Gamma and IG Lévy densities that these corresponding Lévy processes are special cases of the CMY-process; take $Y=0$ and $Y=1 / 2$ respectitvely.

Clearly, this characteristic function is infinitely divisible. The CMY-process $C=\left\{C_{t}, t \geq 0\right\}$ with parameters $C, M>0$ and $Y<1$ is defined as the stochastic process which starts at zero and has stationary, independent CMYdistributed increments. More precisely, the time enters in the first parameter: $C_{t}$ follows a $\operatorname{CMY}(C t, M, Y)$ distribution.

The properties of the $\operatorname{CMY}(C, M, Y)$ distribution given in Table 3 can easily be derived from the characteristic function.

\begin{tabular}{|c|c|}
\hline & $\mathrm{CMY}(C, M, Y)$ \\
\hline mean & $C M^{Y-1} \Gamma(1-Y)$ \\
variance & $C M^{Y-2} \Gamma(2-Y)$ \\
skewness & $\frac{C M^{Y-3} \Gamma(3-Y)}{\left(C M^{Y-2} \Gamma(2-Y)\right)^{3 / 2}}$ \\
kurtosis & $3+\frac{C M^{Y-4} \Gamma(4-Y)}{\left(C M^{Y-2} \Gamma(2-Y)\right)^{2}}$ \\
\hline
\end{tabular}

Table 3: Mean, variance, skewness and kurtosis of the CMY $(\mathrm{C}, \mathrm{M}, \mathrm{Y})$ distribution.

Let us start with a CMY-process $C=\left\{C_{t}, t \geq 0\right\}$ with parameters $C, M>0$ and $Y<1$. As driving Lévy process (in a risk neutral setting), we then take:

$$
X_{t}=\mu t-C_{t}, \quad t \in[0,1],
$$

where in this case $\mu=r-\log (\phi(-i))=r-C \Gamma(-Y)\left((M+1)^{Y}-M^{Y}\right)$. We hence have a deterministic up-trend and negative shock are coming from a CMYprocess. We refer to this model as the Shifted CMY model (SCMY).

The characteristic exponent in this case is available in closed form. More precisely,

$$
\psi(z)=\mu z+C \Gamma(-Y)\left((M+z)^{Y}-M^{Y}\right) .
$$

Note further that

$$
h(\lambda)=\lambda+C \Gamma(-Y)\left(\left(M+\lambda \mu^{-1}\right)^{Y}-M^{Y}\right) \text { and } h^{-1}\left(M+\lambda \mu^{-1}\right)^{Y-1} .
$$




\section{Pricing of Credit Default Swaps}

Consider a CDS with maturity $T$ and a continuous spread $c$. The price of a this CDS is then given by

$$
C D S=(1-R)\left(-\int_{0}^{T} \exp (-r s) \mathrm{d} P(s)\right)-c \int_{0}^{T} \exp (-r s) P(s) \mathrm{d} s,
$$

where $R$ is the asset specific recovery rate and $r$ is the default-free discount rate.

From this, we find the par spread $c^{*}$ that makes the CDS price equal to zero:

$$
c^{*}=\frac{(1-R)\left(-\int_{0}^{T} \exp (-r s) \mathrm{d} P(s)\right)}{\int_{0}^{T} \exp (-r s) P(s) \mathrm{d} s}
$$

\subsection{Goodness of Fit}

We calibrated the different models to a whole family of CDS curves. In the calibration, we minimized the absolute error between model CDS quotes and market CDS quotes. We have weekly data from all the 125 companies on the iTtraxx over the year 2005. So, we have 125 CDS curves for 52 weeks, making in total 6500 CDS curves with spread rates of $1,3,5,7$ and 10 year maturities. In Table 4, one finds a comparison of the mean error over the 125 components for the different maturities together with the total error and the average error per quote for the calibration on the data set of the 5th of January 2005.

\begin{tabular}{llllllll}
\hline \hline Model & 1y & 3y & 5y & $\mathbf{7 y}$ & $\mathbf{1 0 y}$ & total & $\begin{array}{l}\text { average } \\
\text { per quote }\end{array}$ \\
\hline SG & 3.2088 & 0.6302 & 1.4056 & 0.0274 & 2.9150 & 8.1870 & 1.6374 \\
SIG & 2.8384 & 0.5689 & 1.3062 & 0.0280 & 2.7926 & 7.5342 & 1.5068 \\
SCMY & 1.4187 & 0.2203 & 0.6710 & 0.2262 & 1.4836 & 4.0199 & 0.8040 \\
\hline \hline
\end{tabular}

Table 4: Mean absolute error (in bp) for calibration of different models on the 125 CDS in iTtraxx on 5th of January 2005

In Figure 1, one sees the fit obtained under the the different models for company Electrolux $\mathrm{AB}$ on that day (o-signs are market prices and + -signs are calibrated model prices).

One observes, that the SCMY model outperforms the SG and SIG, but that also for the two latter models the fit is very acceptable.

In Figure 2, one sees a histogram of the absolute errors over the 125 CDS curves calibration on one particular day under SCMY, namely on the 5th of January 2005. One sees, that the majority of the 125 companies have an average error per quote less than $1 \mathrm{bp}$.

Next, we will look to the time-evolution of the fit. In Figure 3, one sees a the evolution of the average errors per quote over the 52 weeks for the SIG, SCMY 
model and the SG model. As expected SCMY outperforms SG and SIG. For the SCMY model, one can find the evolution of the mean absolute error over time for each maturity in Figure 4.

\subsection{Discussion of CDS Calibration Results}

Next, we take a closer look at the calibration results. We focus on the CMY model, because the two other models, Gamma and IG, are subcases of the CMY model. We have estimated the three parameters, $C, M$ and $Y$ for each of the 125 underlying CDS curves for each of the 52 weeks.

The estimation is done under risk-neutral measure and hence the interpretation of the Lévy measure is the market prices moves of different sizes. Since, the Lévy measure is parameterized by three parameters, one may relative freely estimate the prices of moves at three different size magnitudes. We therefore focus attention on the 5, 10 and 20 percentage moves in asset value. We report the approximate daily arrival rates of a 5 percent move and call this variable the level of the market. In addition, we report the premium of 5 percent moves to 10 percent moves measured by the ratio of the Lévy measure at 10 percent to that of 5 percent and call this the 10 to 5 percent market spread. Finally, we report the relative spread, defined as the logarithm of the 10 to 5 percent market spread over the 20 to 10 percent market spread. These statistics are computed as a one-to-one transformation of the parameters for each underlier and for each date. We then average across all underliers at each date. Figure 5 presents a graph of the level, spread and relative spread together with the mean of the 5 year cds quote over the year averaged over all names.

We observe that the level of 5 percent moves is just below one move per week. Though this is high from physical measure viewpoint, the pricing is risk-neutral and the market prices in a greater degree such moves. The level of down-moves follows closely to the pattern of the 5 year CDS quote. The spread of 10 percent to 5 percent move is fairly stable around 20 percent and so the market prices in

a 10 percent move every 5 weeks. We note however, that the structure of these negatives moves is compensated by the positive drift. We observe that there is a negative correlation between the mean cds and the relative spread. This suggests that if CDS rates rise the prices of 5 percent moves follow, but prices for larger moves like 20 percent remains relatively stable.

\section{References}

[1] J. Bertoin, Lévy Processes , Cambridge Tracts in Mathematics 121, Cambridge University Press, Cambridge, 1996.

[2] F. Black, and J. Cox, Valuing corporate securities: some effects on bond indenture provisions. J. Finance, 31(1976), pp. 351-367.

[3] J. Cariboni, and W. Schoutens, Pricing Credit Default Swaps under Lévy Models. Journal of Computational Finance, 10(4)(2007). 
[4] A.E. Kyprianou (2006) Introductory Lectures on Fluctuations of Lévy Processes with Applications, Springer.

[5] L.C.G. Rogers, Evaluating first-passage probabilities for spectrally onesided Lévy processes. J. Appl. Probability 37(2000), 1173-1180.

[6] K. Sato, Lévy Processes and Infinitely Divisible Distributions. Cambridge Studies in Advanced Mathematics 68, Cambridge University Press, Cambridge, 2000.

[7] W. Schoutens, Lévy Processes in Finance: Pricing Financial Derivatives, Wiley, 2003. 

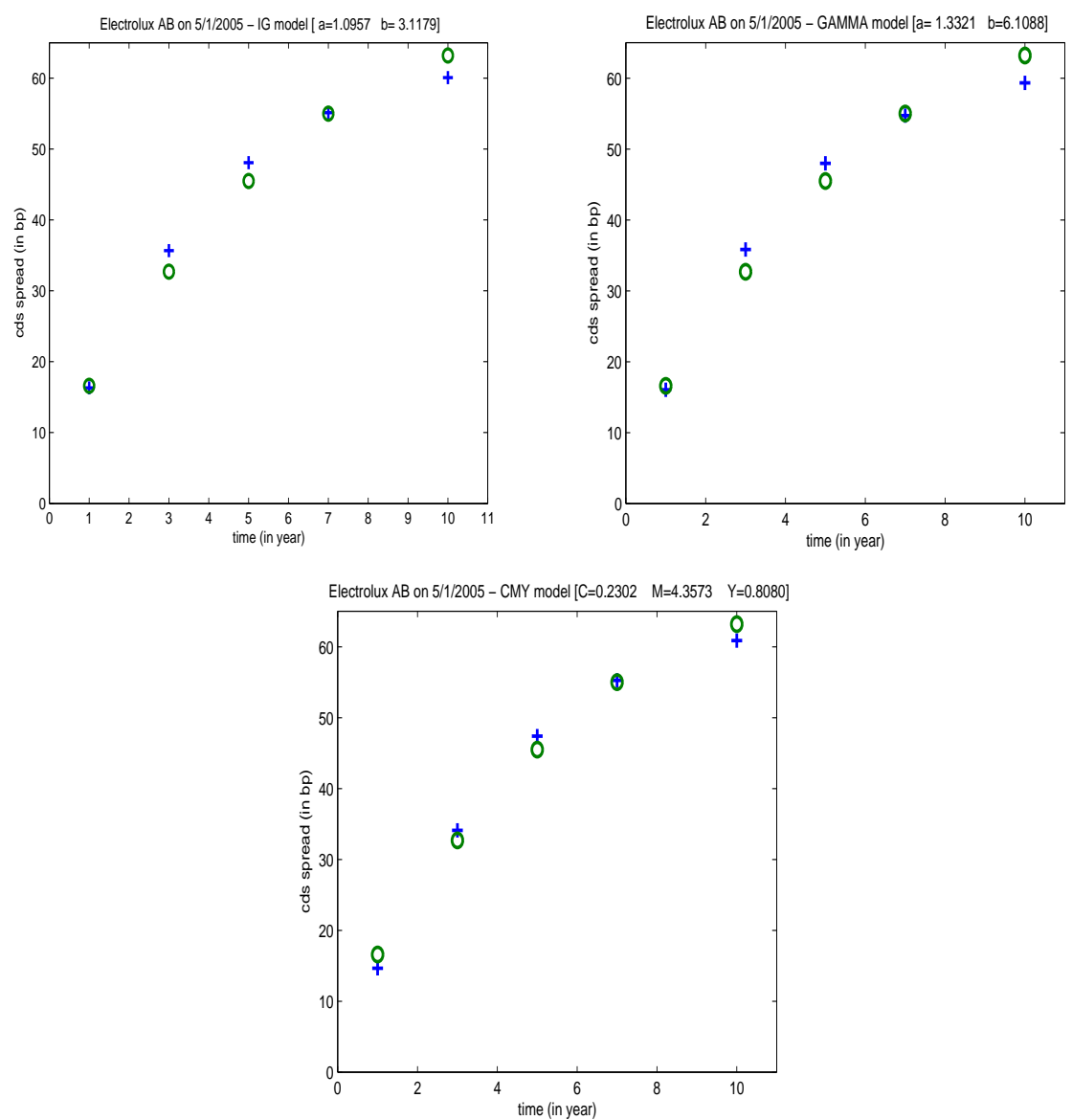

Figure 1: Fit for Electrolux on 5/1/2005 under the IG, GAMMA and CMY model 


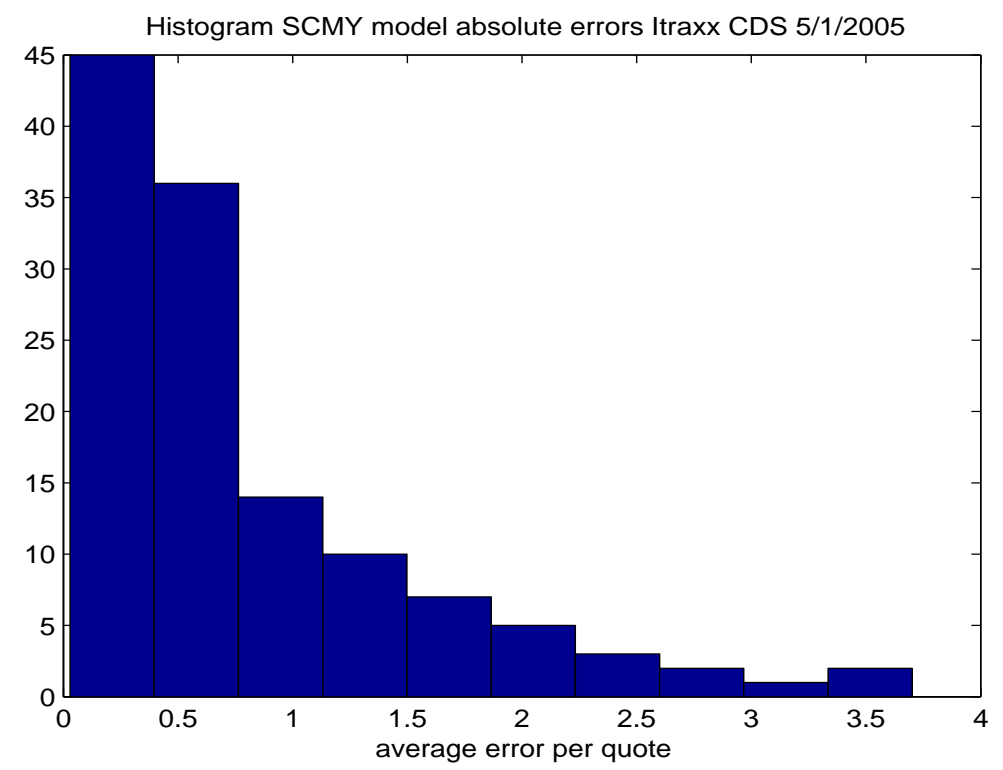

Figure 2: Histogram of calibration error per quote of SCMY on iTraxx on 5Jan-2005 


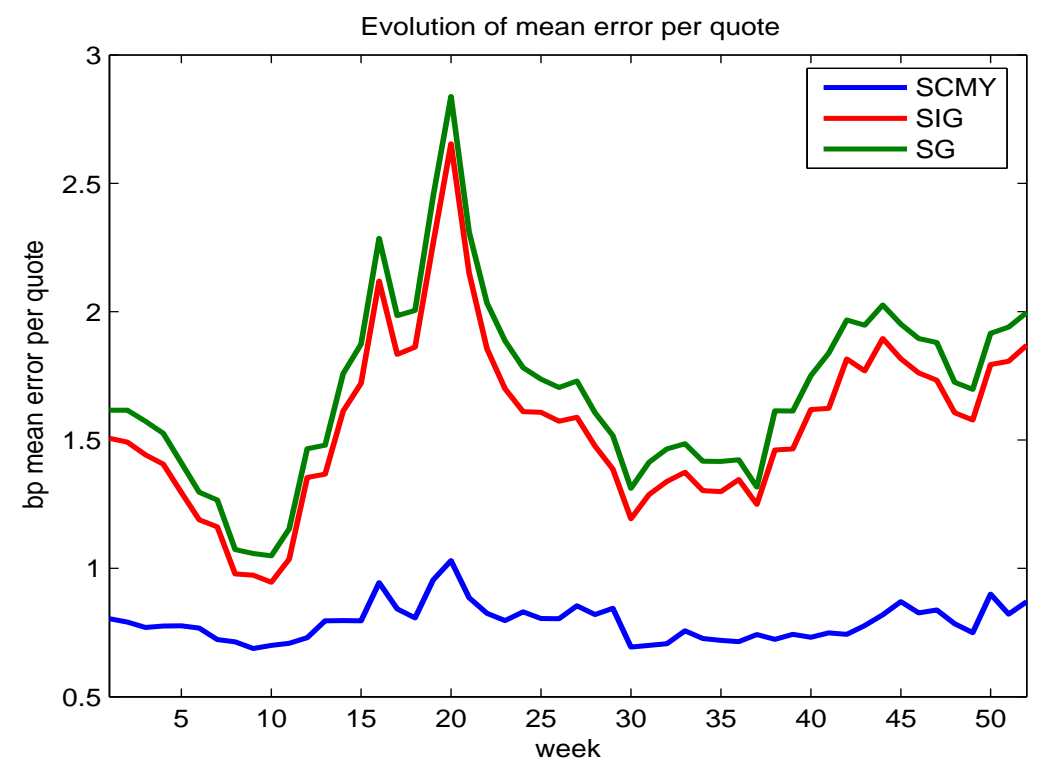

Figure 3: Evolution over time of average calibration error per quote of SG, SIG and SCMY on iTraxx in 2005 


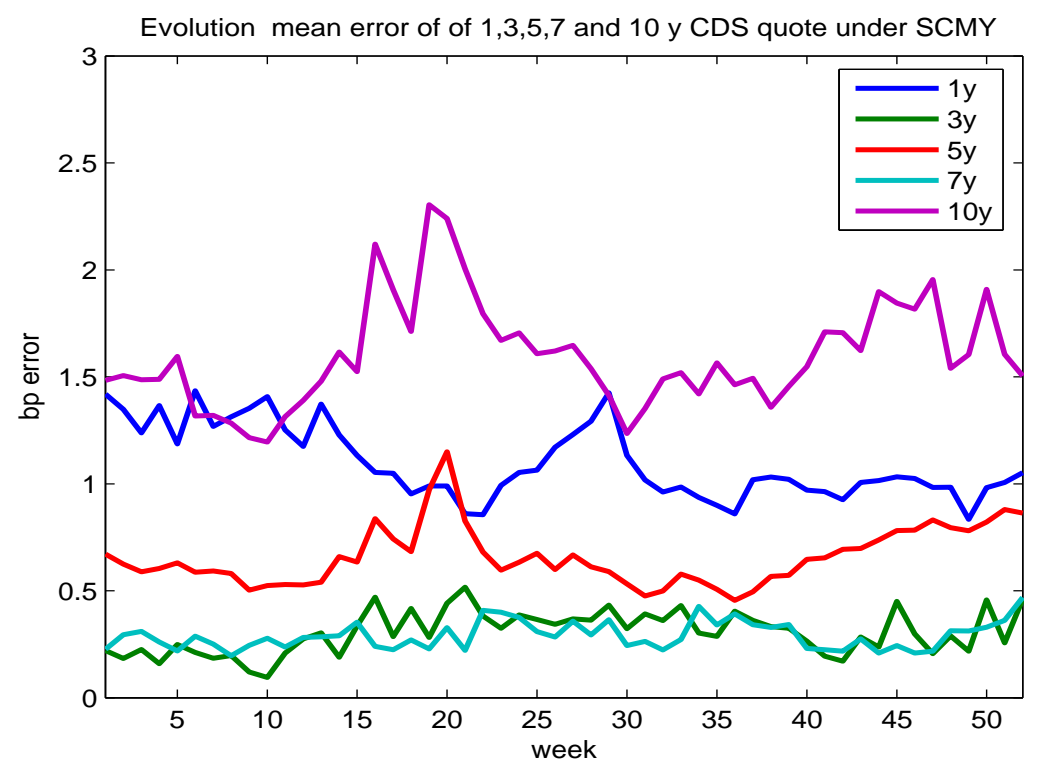

Figure 4: Evolution over time of average calibration error per maturity of SCMY on iTraxx in 2005 

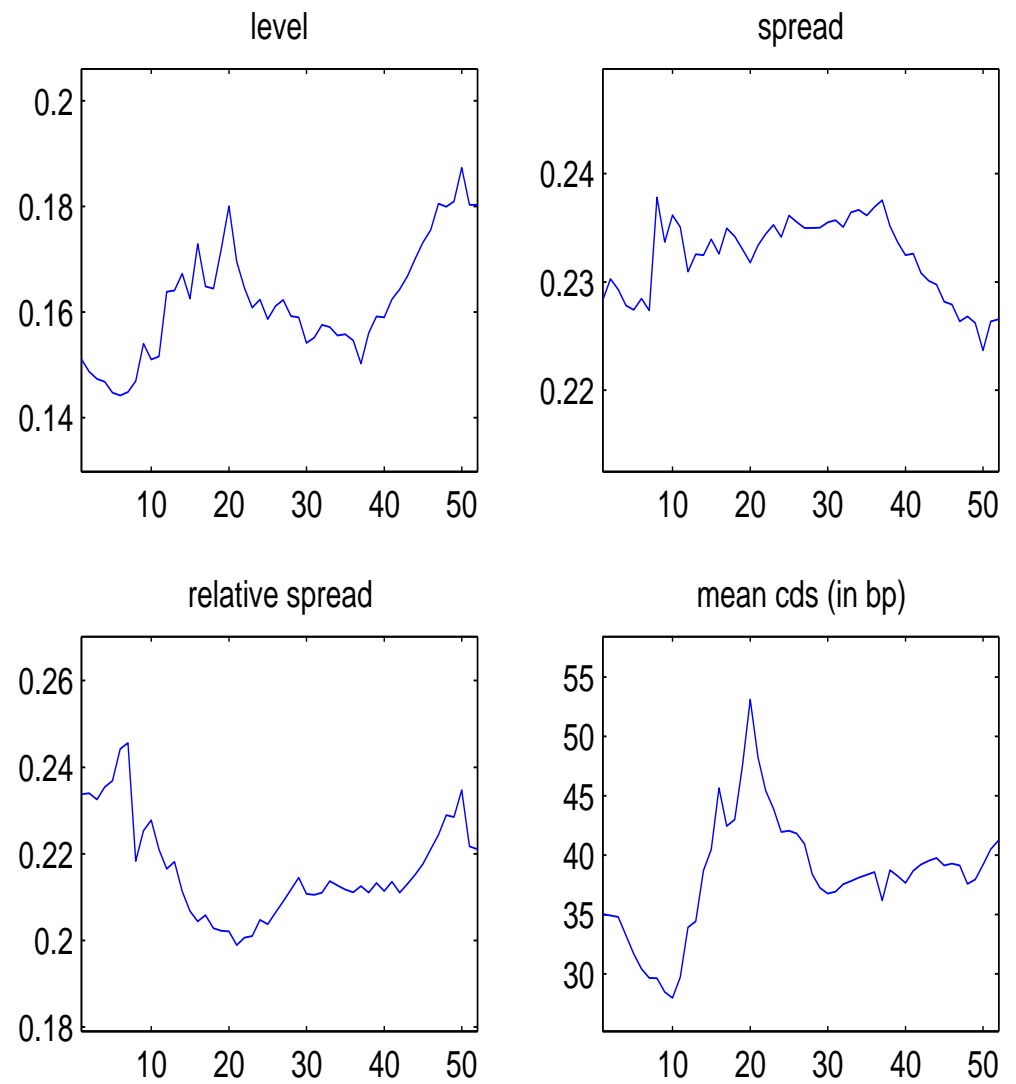

Figure 5: Evolution of level, spread, relative spread and mean cds value under SCMY calibrated model 